\title{
Pas de poésie sans engagement
}

Entretien mené par Julien Jeusette

Lisette Lombé

\section{(2) OpenEdition}

12 Journals

Édition électronique

URL : https://journals.openedition.org/coma/5916

DOI : $10.4000 /$ coma.5916

ISSN : 2275-1742

Éditeur

Institut des textes \& manuscrits modernes (ITEM)

Référence électronique

Lisette Lombé, "Pas de poésie sans engagement », Continents manuscrits [En ligne], 15 | 2020, mis en ligne le 15 octobre 2020, consulté le 13 janvier 2023. URL : http://journals.openedition.org/coma/5916 ; DOI : https://doi.org/10.4000/coma.5916

Ce document a été généré automatiquement le 13 janvier 2023.

\section{(c) (i) (2)}

Creative Commons - Attribution - Pas d'Utilisation Commerciale - Pas de Modification 4.0 International - CC BY-NC-ND 4.0

https://creativecommons.org/licenses/by-nc-nd/4.0/ 


\title{
Pas de poésie sans engagement
}

\author{
Entretien mené par Julien Jeusette
}

Lisette Lombé

\section{NOTE DE L'ÉDITEUR}

Artiste plurielle, passe-frontières, LISETTE LOMBÉ (1978) déploie son art à travers des pratiques poétiques, scéniques, plastiques, militantes et pédagogiques. Dans ses divers espaces d'écriture et de luttes, elle insiste sur sa propre chair métissée, son parcours de femme, de mère et d'enseignante. De ces engagements multiples où l'intime est politique et la parole performative dérivent des collages, des spectacles, des livres et des ateliers, " passeurs de rage et d'éros » comme elle l'écrit. Elle a mené des ateliers d'écritures dans le monde entier, de l'Irak au Congo en passant le Maroc et Haïti. Cofondatrice du collectif L-SLAM, elle a été récompensée, en 2017, du titre de Citoyenne d'Honneur de la Ville de Liège pour sa démarche d'artiviste et d'ambassadrice du slam aux quatre coins de la Francophonie. Elle a publié, entre autres, La Magie du Burn-out (Image Publique, 2017), Black Words (L'Arbre à Paroles, 2018), On ne s'excuse de rien (Maelström ReÉvolution, 2019), Tenir (Maelström Reévolution, 2019), Venus Poetica (L'Arbre à Paroles, 2020), Brûler, brûler, brûler (Iconoclaste, 2020). En 2015, elle a obtenu la seconde place au prix Paroles Urbaines, dans la catégorie «slam ». 


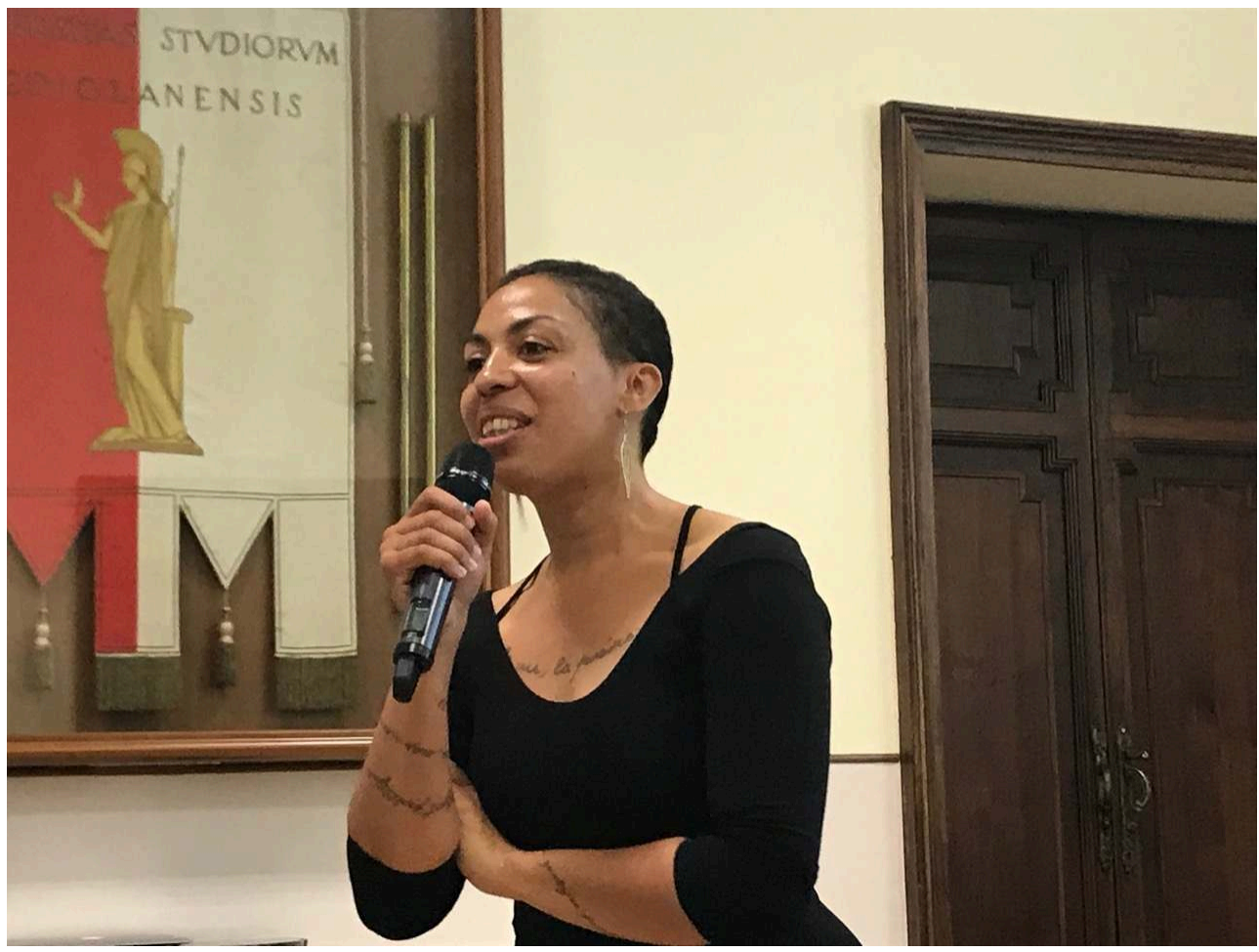

Photo de Silvia Riva

JULIEN JEUSETte : «Elle n'oublie pas qu'elle vient de loin», peut-on lire sur votre site internet. Qu'est-ce que cela signifie pour vous? À l'heure où les écrivains d'origine congolaise vivent éparpillés à travers le monde, considérez-vous vous qu'il existe quelque chose comme une «littérature congolaise» aujourd'hui? Si oui, est-ce qu'elle vous intéresse plus qu'une autre?

Lisette Lombé : En réalité, le territoire lointain que j'évoque dans ma présentation fait moins référence à mes origines congolaises qu'à mes précédentes expériences professionnelles en lien avec l'enseignement et la formation. Je n'étais pas prédestinée à mener une carrière artistique, j'ai découvert de manière fulgurante les réalités des arts de la scène et j'ai dû faire le deuil du salariat pour un statut d'indépendante. Néanmoins, en tant que femme métisse, ma couleur de peau m'assigne à ce territoire lointain, comme si je portais le Congo de mon père sur mon visage, comme si ce Congo me précédait dans tout échange. Je suis née à Namur, je suis belge, mais je reste perçue comme étrangère, non blanche, non européenne.

Hier, j'étais invitée dans une librairie bruxelloise spécialisée en afro-littératures, "Pépite Blues», et en regardant les rayonnages, je pouvais voir se dessiner une communauté d'autrices et d'auteurs, du continent ou de la diaspora, une famille aux contours fluides, mélange d'Africains et d'Afro-européens. Pourquoi pourrait-on parler de littérature sénégalaise ou haïtienne, par exemple, et pas de littérature congolaise? Par contre, je mentirais en affirmant que mes origines congolaises me portent plus particulièrement vers des lectures d'écrivains et écrivaines congolaises. J'aime la littérature, sans frontières, et en particulier les essais et la poésie de tous les pays.

J. J. : Dans votre recueil Black Words, vous démontez notamment les stéréotypes d'une certaine représentation de la femme africaine. Considérez-vous que votre écriture est 
engagée, militante, impliquée ? Comment reliez-vous l'écriture poétique à votre engagement intellectuel (votre intérêt pour l'éducation populaire, les ateliers d'« empowerment » pour les femmes)?

L. L. : Dans la postface de Black Words, je dis que « si on entend par engagée le fait que je transforme en poèmes un sentiment de révolte face aux discriminations, que j'assume chacun des mots que j'écris et que je partage ceux-ci dans le but de faire bouger des lignes dans les imaginaires collectifs, alors oui, je suis une auteure engagée. Le je que j'utilise dans mes textes renvoie toujours à un nous parce que je suis consciente que ma présence sur scène, en tant que femme racisée, métisse, mère de famille, peut avoir valeur de brèche ou de porte pour d'autres. »

Pour moi, il n'y a pas de possibilité d'épanouissement individuel sans émancipation collective. Pas de scènes sans partage, pas de littérature sans slam, pas d'artistique sans éducation permanente, pas de culturel sans social, pas de démocratie sans paroles citoyennes, pas de poésie sans engagement.

Le focus " empowerwomen » est une réponse à des observations des scènes de slam que je fréquentais. Faire confiance à mon corps de femme et aux dissonances qu'il ressentait. Ne plus me contenter des «Tout qui a écrit un texte peut venir partager son texte!", sans réfléchir aux freins qui empêchent certaines personnes de parvenir jusqu'à cet espace d'expression démocratique. En 2015, dans le public, plus d'hommes que de femmes. Sur scène, plus d'hommes que de femmes. Pourquoi ces dernières disparaissent-elles entre les ateliers d'écriture et ces soirées, entre ces soirées et les concours? Où sont les femmes racisées, les moins valides, les personnes trans, les mamans solos, celles qui portent le voile? Toutes ces questions deviendront le terreau d'une méthodologie propre à mon association L-SLAM.

J. J. : Le recueil de poésie n'est qu'un mode parmi d'autres de votre travail artistique : vous pratiquez le slam, le collage, l'installation, la «conférence gesticulée »... Y a-t-il un point commun entre ces différentes formes artistiques? Comment les reliez-vous? Et enfin, quelle place donnez-vous au recueil de poésie aujourd'hui, a-t-il encore un rôle à jouer à I'heure d'Internet, du rap, du slam (qui refuse souvent la publication)?

L. L : Le fait d'être métisse, ni blanche ni noire, et blanche et noire, influence ma vie, ma pratique artistique et mon militantisme. Tout est lié : défendre l'intersectionnalité, affectionner les parcours chaotiques, les entre-deux et décloisonner les genres. On ne peut pas prôner un accès plus démocratique à la culture et dans le même temps proposer une poésie hermétique donc élitiste. Avec le slam, il s'agit de court-circuiter le temps long de l'édition, le circuit fermé de l'écrit et des institutions. La danse, le collage, les installations de street art me permettent d'enrichir mes mots d'une dimension visuelle, plus inclusive.

Pour nous, poètes performeurs, l'objet-livre n'est jamais synonyme de la naissance de notre poésie, mais est la synthèse joyeuse de plusieurs mois de partage et d'évolution de nos textes sur scène. Être édité reste une cerise sur le gâteau, un cadeau inattendu, non attendu. Je suis consciente de ma chance : après chaque scène, chaque atelier, je peux vendre des livres. Grâce et à cause de la scène, je suis, à la fois, l'ambassadrice, la femme-sandwich et la chargée de diffusion de ma littérature. Si je n'étais pas une slameuse, je vendrais beaucoup moins de livres. Je constate que le grand public aime prolonger le voyage proposé sur scène ou en atelier par des lectures, un retour à l'intime, à la résonnance et au silence. Le papier reste important pour sa texture, sa trace, sa longévité, sa charge historique et fantasmatique. Les éditions Maelström ont 
une collection de booklegs vendus à trois euros qui cartonne, car elle répond à la fois au goût du livre et aux goûts du temps.

J. J. : La «world literature » et la sociologie de la littérature pensent souvent le rapport de l'écrivain à l'écriture en termes de "stratégie». Ce terme vous semble-t-il adéquat pour envisager la manière dont un écrivain ou un artiste bâtit son œuvre?

L. L. : Si stratégie il y a, ce n'est qu'a posteriori. Selon moi, on écrit par impériosité d'écrire. Quelque chose doit se dire, doit sortir, doit noircir la page. Ensuite, pour des questions de survie même, et de l'écrivain.e et de la famille ou de l'industrie qui l'entoure, il faudra effectivement faire preuve de pragmatisme. Dans quelle langue le livre se vendra-t-il le mieux? Dans quel rayon, sous quelle étiquette? Je suis poétesse. La poésie, en termes de vente, reste le parent pauvre de la littérature. Je ne me suis pas levée un matin en me disant que, pour mieux gagner ma vie, je devrais plutôt écrire un roman qu'un recueil de poèmes, mais il se fait que mon dernier ouvrage, Venus Poetica, s'apparente plus à un récit qu'à des textes de slam. Du coup, avec mon éditeur, nous avons longuement discuté de la manière de positionner cette nouvelle facette de mon travail artistique. Annoncer un premier roman érotique semble plus vendeur que de communiquer sur un second recueil de poésie. C'est ce que l'écriture peut concéder à la stratégie.

\section{J. J. : Comment voyez-vous l'avenir de la poésie et de la littérature africaine?}

L. L. : À l'image de l'avenir des luttes et des mouvements qui interrogent et remettent en question l'héritage colonial et patriarcal : de plus en plus visibles, de plus en plus légitimes, de plus en plus populaires, de plus en plus accessibles. Le Vieux Monde prend l'eau pour laisser place à de nouvelles voix. Ceux qui refusent cette réalité appartiennent déjà à un autre temps.

\section{INDEX}

Mots-clés : littérature congolaise, arts de la scène, slam, performance, poésie

\section{AUTEUR}

\section{LISETTE LOMBÉ}

Slameuse, poète, performeuse 